\title{
La formación de futuros docentes de Música en las universidades de Castilla y León: creatividad, ciudadanía y aprendizaje permanente como claves del cambio educativo
}

\author{
Music teacher training at the universities of Castilla y León: creativity, citizenship, and \\ lifelong learning as keys to educational change
}

\author{
Yurima Blanco García \\ yurima.blanco.garcia@uva.es \\ Departamento de Didáctica de la Expresión Musical, Plástica y Corporal \\ Universidad de Valladolid \\ Valladolid, España \\ ORCID: https://orcid.org/0000-0002-4890-7045

$\begin{array}{r}\text { Alicia Peñalba Acitores } \\ \text { alicia.penalba@uva.es }\end{array}$
Departamento de Didáctica de la Expresión Musical, Plástica y Corporal
Universidad de Valladolid
Valladolid, España

doi: 10.7203/LEEME.46.17756

Recibido: 24-06-2020 Aceptado: 03-08-2020. Contacto y correspondencia: Yurima Blanco García. Departamento de Didáctica de la Expresión Musical, Plástica y Corporal, Universidad de Valladolid, Campus La Yutera, Avenida de Madrid 50, C.P. 34004, Palencia. España.

\section{Resumen}

El presente artículo analiza la formación musical de los futuros docentes de música de las enseñanzas obligatorias en las universidades de Castilla y León. Forma parte de un proyecto I+D EDU2017-84782 (Profmus) que investiga sobre la formación del profesorado y su impacto en la actual política educativa. Se examinan 8 planes de estudio ( 5 de Grado y 3 de Máster) y un total de 70 asignaturas: 45 del Grado de Maestro/a (Mención en Educación Musical), y 25 del Máster de Profesorado de Enseñanza Secundaria Obligatoria. Se analizan los contenidos de las memorias de verificación, guías docentes y legislación de la educación obligatoria a través de codificación abierta. Se encuentran ocho categorías de intersección entre los planes universitarios y el currículo de Primaria y Secundaria, en dos dimensiones: contenidos musicales (escucha; interpretación musical; danza y movimiento; contextos musicales y culturales; música y tecnologías) y contenidos transversales (expresión oral y escrita; emprendimiento y creatividad; ciudadanía y diversidad). Los resultados muestran que por lo general los planes de estudio se adecúan a las necesidades formativas de los futuros docentes, aunque se requiere orientar la formación hacia la creatividad, ciudadanía y aprendizaje a lo largo de la vida.

Palabras clave: Formación de profesorado de música; currículum; Educación Primaria; Educación Secundaria Obligatoria; Universidad.

\section{Abstract}

This article analyses the musical training of future music teachers in compulsory education at the universities of Castilla y León. It is part of an R+D project EDU2017-84782 (Profmus) that researches the training of teachers and its impact on current educational policy. It examines eight courses of study (five Bachelor's and three Master's) and a total of 70 courses: 45 from the Teacher's Degree (Music Education), and 25 from the Master's Degree in Compulsory Secondary Education. The curricula, teaching guides and compulsory education legislation are analyzed through open coding. Eight categories of intersection between university plans and the primary and secondary curriculum are found, in two dimensions: musical content (listening, musical interpretation, dance and movement, musical and cultural contexts, music and technologies) and transversal content (oral and written expression, entrepreneurship and creativity, citizenship and diversity). The results show that courses of study are generally adapted to the training needs of future teachers, although training needs to be oriented towards creativity, citizenship, and lifelong learning.

Key words: Music Teacher training; curriculum; Primary Education; Secondary Education; University. 


\section{Introducción}

La educación musical no es ajena a las transformaciones y los retos que asumen los sistemas educativos en la actualidad. La educación concebida como motor económico del conocimiento menoscaba, a corto plazo, las necesidades culturales y el pensamiento holístico que demanda la sociedad del conocimiento (Aróstegui, 2006). Desde una perspectiva crítica, se observa un declive de la educación musical, reducida en el actual currículo de la enseñanza obligatoria. La disminución de horas de Educación Artística en la jornada escolar, el carácter optativo de sus asignaturas y la mayor ventaja de las denominadas áreas STEM (Ciencia, Tecnología, Ingeniería y Matemáticas) indican un "claro retroceso" para la formación artística, ciudadana e integral de los educandos, hecho que se contextualiza no sólo en España, sino que se extiende a otros países (Aróstegui, Louro y Teixeira, 2015).

La formación inicial del profesorado de Música en la Educación Primaria y Secundaria resulta clave para afrontar los desafíos de este cambio educativo. En el nuevo marco legislativo conviene indagar sobre los planes de estudios que rigen la formación musical de los futuros docentes, valorar la adecuación respecto a las necesidades que se plantean en las aulas y su preparación para dar respuesta a las demandas individuales, sociales, culturales y económicas en el contexto de la sociedad y la economía del conocimiento (Profmus, 2017).

Este propósito se enmarca en un proyecto de investigación de alcance nacional, que pretende aportar conocimiento sobre la formación inicial y permanente del profesorado de música en España, documentar experiencias docentes y examinar su impacto en la actual política educativa (Profmus, 2017). La relevancia de esta investigación se muestra también en otras regiones geográficas, como el proyecto Music Teacher Education for the Future (Futured, 2019), en Noruega, o las investigaciones de Colleen Conway en la Universidad de Michigan, cuyo principal resultado es un Manual sobre Formación Inicial del Profesorado de Música (Conway, Pellegrino, Stanley y West, 2019) para su aplicación en Estados Unidos. Todos estos proyectos comparten una visión crítica sobre la formación de los futuros docentes de música y su adecuación a los contextos actuales.

En consonancia con dichos proyectos, en el presente artículo se examina la formación inicial que recibe el profesorado de música en el marco de las universidades de Castilla y León, para lo cual se analizan los planes de estudios y su articulación con la legislación vigente en las enseñanzas obligatorias. Interesa conocer cómo se estructuran estos planes, cómo se articulan respecto a los contenidos del actual currículo de Educación Primaria y Secundaria y en qué medida responden a las necesidades de los educandos y de los contextos culturales, sociales y económicos donde se desarrollan. 


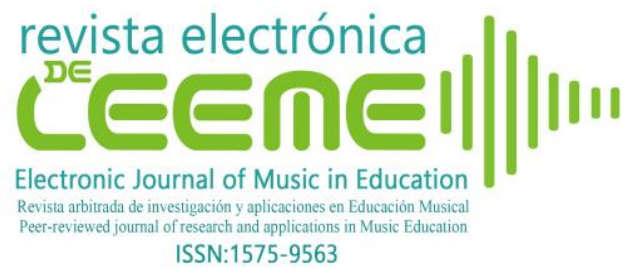

\subsection{Revisión teórica}

El proceso de convergencia europea de Educación Superior ha conllevado importantes cambios en la formación del profesorado de Música. Entre las características del nuevo modelo, destacan la estructuración en dos ciclos, el sistema de equivalencias entre las titulaciones y el aprendizaje basado en competencias. Aróstegui (2006) señala entre sus consecuencias un mayor énfasis en la consecución de objetivos mensurables y productivos, antes que "capacidades interpretativas y estéticas del ser humano" (p.842). Al respecto, plantea la necesidad de contextualizar los procesos de enseñanza; asumir un modelo de currículum crítico ajustado a las necesidades de cada realidad y apropiados al área de conocimiento - en nuestro caso, la educación musical一; y tomar en cuenta "aspectos emocionales, físicos, perceptivos, estéticos y creativos” en la evaluación del profesorado de música (Aróstegui, 2006, p.842).

La red de investigación ALFA desarrolló, entre 2004-2007, una revisión razonada de los planes de estudios de la formación del profesorado de música en América Latina y Europa. Los resultados constataron diferentes aplicaciones y efectos de las políticas de reforma, mediados por el impacto que ejercen los contextos en los procesos de formación (Aróstegui, 2010). Los datos apuntan a la falta de unanimidad en los criterios de convergencia: en la estructura (ciclos de $3+2$ y de $4+1$ ); gran disparidad en cuanto a los contenidos; distinto énfasis en el perfil profesional, ya sea hacia una formación generalista (donde el docente imparte, entre otras asignaturas, música) o, hacia una mayor especialización (Aróstegui y Cisneros-Cohernour, 2010). Se precisa, entonces, de un modelo consolidado y la necesidad de formar maestros de música - antes que "músicos educadores" —, preparados para dar respuesta a las necesidades de la Educación Obligatoria (p.189).

En España, la formación del profesorado especialista de música se rige por el Real Decreto 1393/2007, de 29 de octubre, por el que se establece la ordenación de las enseñanzas universitarias oficiales (Ministerio de Educación y Ciencia, 2007b). Formación musical es parte del módulo didáctico y disciplinar del Grado de Maestro/a en Educación Primaria, incluida en el apartado "Educación Musical, Plástica y Visual". Las competencias previstas comprenden, entre otras, el conocimiento del currículo escolar, la adquisición de recursos didácticos y el desarrollo de actividades para la participación artística dentro y fuera de la escuela (Ministerio de Educación y Ciencia, 2007a). En relación con el profesorado de Música de Educación Secundaria Obligatoria (ESO, en adelante), su habilitación comprende los estudios del Máster de Profesor de Educación Secundaria Obligatoria (ESO) y Bachillerato, Formación Profesional y Enseñanza de Idiomas, con la especialidad correspondiente (Ministerio de Educación, 2008).

Una reflexión constante sobre la formación del profesorado es si se sirve mejor la educación musical escolar con maestros generalistas o especializados (Aróstegui y Kyakuwa, 2020). Actualmente, coexisten docentes de Música con diversas titulaciones en las escuelas, 


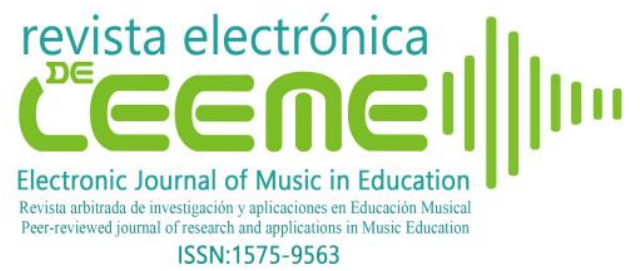

como los formados en los extintos programas de diplomatura en Educación Musical o los graduados de Maestro/as en Educación Primaria, según el plan vigente. Este último presenta una peculiaridad: es maestro/a generalista, ya que ha adquirido las competencias en todas las áreas del título y, a la vez, posee una mención cualificadora como especialista en Educación Musical. Sin embargo, según algunos autores (López-García, Madrid y De Moya, 2017) esta modalidad no parece suficiente, en tanto supone una reducción considerable de créditos dedicados a la formación técnico-musical, limitados a poco más de 30 ECTS específicos de Mención. Otra de las limitaciones radica en la ausencia de una prueba de acceso y, en consecuencia, un alumnado heterogéneo en su preparación musical, una de las mayores dificultades en la especialización (Reyes-López, 2010).

Diferentes estudios examinan la formación musical que reciben los futuros docentes en España, con diversos enfoques y puntos de atención: respecto al perfil profesional y las competencias deseables en el futuro profesorado de música (Carrillo y Vilar, 2014); su perfil sociocultural (Mateos-Moreno, 2013); la práctica educativa y el aprendizaje servicio (Gillanders, Torres y Pérez, 2018); el uso e integración de las TICs en los planes docentes (Calderón, Gustems, y Carrera, 2020); la percepción de los futuros maestros sobre el aprendizaje musical y sus carencias (Rosa-Napal, Muñoz-Carril, González-Sanmamed y Romero Tabeayo, 2020) o a través de una perspectiva comparada con otros países (Ivanova y Siebenaler, 2018; Aróstegui y Kyakuwa, 2020). Sin embargo, apenas se localizan estudios generalizados que evalúen los planes actuales, aunque se cuenta con antecedentes, como el mencionado proyecto ALFA, enmarcado en el tránsito hacia el EEES. Otra referencia se sitúa en el proyecto IMPACTMUS que, si bien no evalúa el currículo de la formación de profesorado, examina el impacto de la música en docentes, alumnado e instituciones de las enseñanzas obligatorias ${ }^{1}$.

Serrano y Peñalba (2019) señalan que la adecuada formación del profesorado resulta fundamental para encauzar la educación musical en la escuela y que las características del profesor/a tendrán un impacto -positivo o negativo- en la recepción de la música en el aula. Estas características no se reducen a habilidades técnicas o artísticas, sino que comprenden "los ámbitos personal, pedagógico y musical" conducentes al perfil de un buen docente de música (p.112). En esa línea, Domínguez (2014) discute sobre la necesidad de formar a los docentes de música desde la perspectiva de ciudadanos críticos, que les permita desarrollar en las aulas la creatividad, el trabajo cooperativo, el emprendimiento, entre otras capacidades necesarias para afrontar los retos de la sociedad actual. Asimismo, Elliott y Silverman (2019) proponen sustentar el currículum en la formación de valores éticos, que fomenten en el profesorado el

\footnotetext{
1 "El impacto de la educación musical en la sociedad y en la economía del conocimiento" (2015-2018), con referencia EDU2014-58066-P, financiado por el Ministerio de Economía y Competitividad de España. Véanse las monografías editadas por Pérez-Moreno y Carrillo (2019) y Aróstegui (en prensa).
}

@Yurima Blanco García y Alicia Peñalba Acitores. The content of this article is the sole responsibility of the authors. The Revista Electrónica de LEEME and Universitat de València are not liable for any legal actions that may arise involving the article's content. Revista Electrónica de LEEME - Lista Electrónica Europea de Música en la Educación-. http://ojs.uv.es/index/php/LEEME/index ISSN: 1575-9563. Editores: Universidad de Valencia y Jesús Tejada. Visibilidad de esta revista: SCOPUS, Emerging Sources Citation Index (Clarivate), EBSCO, CINDOC (CSIC), Citefactor, COPAC, Dialnet, DICE (CSIC), DOAJ, e-revistas (CSIC), EBSCO Premier, ERIH+, Gale Cengage Learning, IN-RECS, IRESIE, LATINDEX, MIAR, OCLC Worldcat, RESH, REDIB, RILM Core Journals, SUDOC, ULRICHS. Esta revista es de acceso libre mediante licencia Creative Commons 4.0 CC by. Política de archivado: etiqueta verde SHERPA-ROMEO. 


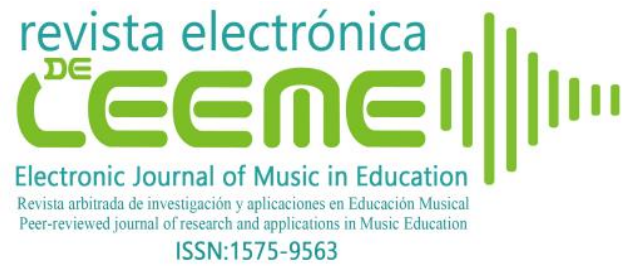

pensamiento, la reflexión y la acción. Estas cualidades concuerdan con algunas necesidades profesionales que se identifican en los futuros docentes.

Todo ello redunda en la necesidad de plantear una formación flexible y crítica, adaptada a las necesidades socioculturales de los distintos contextos, que tome en cuenta las diferentes dimensiones y competencias del currículum, entre otras, tecnológicas (Tejada y Pérez-Gil, 2016); inclusiva y de respeto a la diversidad (Peñalba, 2018); que conceda importancia al patrimonio cultural (Riaño y Cabedo-Mas, 2013), la acción comunitaria y el aprendizajeservicio (Chiva, Salvador, Ferrando y Cabedo-Mas, 2019); y la formación a lo largo de la vida (Cabedo-Mas, 2011).

Tomando en cuenta estas ideas, el objetivo del estudio es conocer si la formación del profesorado de Música en las universidades de Castilla y León se adapta a las necesidades profesionales de los docentes tras las actuales reformas educativas y a las necesidades educativas del alumnado, en consonancia con los cambios sociales y culturales del país.

\section{Método}

El presente estudio tiene una visión prospectiva, en tanto no cuenta con un marco de referencia sobre cómo se desarrollan estos objetivos en el resto del país, aunque sí pone en relación dichos planes de estudios con los currículos de Educación Primaria y de Educación Secundaria Obligatoria (ESO). Se enmarca en el Proyecto I+D Formación del Profesorado y Música en la Sociedad y en la Economía del Conocimiento (Profmus) en el que se estudian, actualmente, los planes de 53 universidades españolas que incluyen estas titulaciones. En ese sentido, la investigación realizada en Castilla y León contribuye al establecimiento de un mapa sobre la formación en Música del futuro profesorado.

Se ha llevado a cabo una revisión de los planes de estudio de las universidades de Castilla y León que imparten la mención de Educación Musical y el Máster en Formación del Profesorado de Educación Secundaria Obligatoria y Bachillerato, Formación Profesional y Enseñanzas de Idiomas y se ha contrastado con las necesidades profesionales impuestas por los currículos de Educación Primaria y de Educación Secundaria. En ese sentido, se adopta un modelo inductivo y de análisis descriptivo de dichas relaciones. Se estudian ocho planes de cinco universidades de Castilla y León: cuatro públicas (Burgos, León, Salamanca y Valladolid) y una privada (Universidad Pontificia de Salamanca): cinco del Grado de Maestro/a en Educación Primaria y tres del Máster de Profesorado. Se analiza un total de 70 asignaturas: 45 del Grado de Educación Primaria y 25 del Máster de Secundaria. A continuación, en las Tablas 1 y 2 , se muestran las asignaturas correspondientes al Grado y Máster. 
Tabla 1. Centros universitarios con formación en Educación Primaria (Mención Música). Asignaturas, cursos y semestres

Universidad

Asignatura

\begin{tabular}{|c|c|c|c|c|}
\hline Universidad & Asignatura & Curso & Semestre & Carácter \\
\hline \multirow{9}{*}{$\begin{array}{c}\text { Universidad } \\
\text { de Valladolid } \\
\text { (UVa) }\end{array}$} & $\begin{array}{l}\text { Fundamentos y Estrategias Didácticas en la Educación } \\
\text { Musical }\end{array}$ & $2^{\circ}$ & 3 & OB \\
\hline & Creación Artística, Cultura Visual y Musical & $3^{\circ}$ & 5 & OB \\
\hline & Formas de Expresión Musical & $3^{\circ}$ & 5 & OP \\
\hline & Música, Cultura y Diversidad & $3^{\circ}$ & 5 & OP \\
\hline & Didáctica de la Expresión Musical & $4^{\circ}$ & 7 & OP \\
\hline & Conjunto Vocal e Instrumental & $4^{\circ}$ & 7 & OP \\
\hline & $\begin{array}{l}\text { Tecnologías de la Información y la Comunicación Aplicadas a } \\
\text { la Educación Musical }\end{array}$ & $4^{\circ}$ & 7 & OP \\
\hline & Prácticum II & $4^{\circ}$ & 8 & $\mathrm{PE}$ \\
\hline & Trabajo Fin de Grado & $4^{\circ}$ & 8 & TFG \\
\hline \multirow{9}{*}{$\begin{array}{l}\text { Universidad } \\
\text { de Burgos } \\
\text { (UBU) }\end{array}$} & Educación Musical & $2^{\circ}$ & 3 & OB \\
\hline & Educación Vocal y Auditiva & $3^{\circ}$ & 5 & OP \\
\hline & Formación Instrumental & $3^{\circ}$ & 4 & OP \\
\hline & Historia de la Música & $3^{\circ}$ & 6 & OP \\
\hline & Agrupación Coral & $4^{\circ}$ & 7 & OP \\
\hline & Composición y Creación Musical & $4^{\circ}$ & 7 & OP \\
\hline & Didáctica de la Expresión Musical & $4^{\circ}$ & 7 & OP \\
\hline & Prácticum II (Educación Musical) & $4^{\circ}$ & 8 & $\mathrm{PE}$ \\
\hline & Trabajo de Fin de Grado & $4^{\circ}$ & 8 & TFG \\
\hline \multirow{10}{*}{$\begin{array}{c}\text { Universidad } \\
\text { de Salamanca } \\
\text { (USAL) }\end{array}$} & Expresión Musical en la Educación Primaria & $2^{\circ}$ & 2 & OB \\
\hline & Formación Vocal & $3^{\circ}$ & 6 & OP \\
\hline & Didáctica de la Expresión Musical & $3^{\circ}$ & 6 & OP \\
\hline & Formación Rítmica y Danza & $4^{\circ}$ & 7 & OP \\
\hline & Formación Instrumental & $4^{\circ}$ & 7 & OP \\
\hline & Creación y Selección de Repertorio Musical para el Aula & $4^{\circ}$ & 7 & OP \\
\hline & Educación Auditiva & $4^{\circ}$ & 7 & OP \\
\hline & Música y Danza para la Diversidad & $4^{\circ}$ & 7 & OP \\
\hline & Prácticum II & $4^{\circ}$ & 8 & PE \\
\hline & TFG & $4^{\circ}$ & 8 & TFG \\
\hline \multirow{6}{*}{$\begin{array}{l}\text { Universidad } \\
\text { Pontificia de } \\
\text { Salamanca } \\
\text { (UPSA) }\end{array}$} & Educación Musical & $3^{\circ}$ & 5 & OB \\
\hline & Lenguaje Musical & $3^{\circ}$ & 5 & OP \\
\hline & Formación Vocal y Auditiva & $3^{\circ}$ & 5 & OP \\
\hline & Didáctica de la Expresión Musical & $4^{\circ}$ & 7 & OP \\
\hline & Historia de la Música & $4^{\circ}$ & 7 & OP \\
\hline & Formación y Agrupación Instrumental & $4^{\circ}$ & 8 & OP \\
\hline
\end{tabular}




\begin{tabular}{clccc}
\hline & Prácticum II & $4^{\circ}$ & 8 & PE \\
& Trabajo Fin de Grado & $4^{\circ}$ & 8 & TFG \\
\hline \multirow{2}{*}{$\begin{array}{c}\text { Educación Musical y su Didáctica } \\
\text { Audición Musical Activa }\end{array}$} & $2^{\circ}$ & 4 & OB \\
& Didáctica de la Expresión Musical & $3^{\circ}$ & 6 & OP \\
de León & Formación Instrumental y Agrupaciones Musicales Escolares & $4^{\circ}$ & 7 & OP \\
& Formación Vocal, Agrupación y Dirección Coral & $4^{\circ}$ & 7 & OP \\
& Formación Rítmica y Danza & $4^{\circ}$ & 7 & OP \\
& Lenguaje Musical & $4^{\circ}$ & 7 & OP \\
& Prácticum II & $4^{\circ}$ & 7 & OP \\
& Trabajo Fin de Grado & $4^{\circ}$ & 8 & PE \\
\hline
\end{tabular}

Fuente: elaboración propia a partir de los datos obtenidos

En cuanto al Máster de Profesorado, se muestra la distribución de las asignaturas en las tres universidades donde se imparte.

Tabla 2. Centros universitarios con formación en Ed. Secundaria: asignaturas, cursos y semestres

\begin{tabular}{|c|c|c|c|c|}
\hline \multirow[t]{7}{*}{ Universidad } & Asignatura & Curso & Semestre & Carácter \\
\hline & Historia de la Música & $1^{\circ}$ & 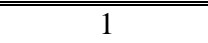 & OB \\
\hline & $\begin{array}{c}\text { Teoría de la Música y Prácticas Musicales } \\
\text { Tradicionales y Populares }\end{array}$ & $1^{\mathrm{o}}$ & 2 & OB \\
\hline & Diseño Curricular en Música & $1^{\mathrm{o}}$ & 2 & $\mathrm{OB}$ \\
\hline & Didáctica de la Música & $1^{\circ}$ & 2 & OB \\
\hline & Metodología y Evaluación en Música & $1^{\mathrm{o}}$ & 2 & $\mathrm{OB}$ \\
\hline & Innovación Docente en Música & $1^{\mathrm{o}}$ & 2 & $\mathrm{OB}$ \\
\hline \multirow{10}{*}{$\begin{array}{l}\text { Universidad de } \\
\text { Valladolid } \\
\text { (UVa) }\end{array}$} & Iniciación a la Investigación Educativa en Música & $1^{\mathrm{o}}$ & 2 & OB \\
\hline & Prácticas Externas & $1^{\mathrm{o}}$ & 2 & $\mathrm{PE}$ \\
\hline & Didáctica de la Especialidad de Música & $1^{\circ}$ & 1 & OP \\
\hline & Recursos en la Especialidad de Música & $1^{\mathrm{o}}$ & 1 & OP \\
\hline & Metodología en la Especialidad de Música & $1^{\circ}$ & 2 & $\mathrm{OP}$ \\
\hline & Evaluación en la Especialidad de Música & $1^{\circ}$ & 2 & $\mathrm{OP}$ \\
\hline & Contenidos en la Especialidad de Música & $1^{\mathrm{o}}$ & 1 & $\mathrm{OP}$ \\
\hline & Historia en la Especialidad de Música & $1^{\mathrm{o}}$ & 1 & OP \\
\hline & Innovación Docente en la Especialidad de Música & $1^{\mathrm{o}}$ & 2 & $\mathrm{OP}$ \\
\hline & Iniciación a la Investigación & $1^{\mathrm{o}}$ & 2 & $\mathrm{OP}$ \\
\hline $\begin{array}{l}\text { Universidad de } \\
\text { Salamanca } \\
\text { (USAL) }\end{array}$ & $\begin{array}{c}\text { Prácticas Externas Obligatorias } \\
\text { TFM }\end{array}$ & $\begin{array}{l}1^{\circ} \\
1^{\circ}\end{array}$ & $\begin{array}{l}\text { no especificado } \\
\text { no especificado }\end{array}$ & $\begin{array}{l}\text { PE } \\
\text { TFM }\end{array}$ \\
\hline
\end{tabular}




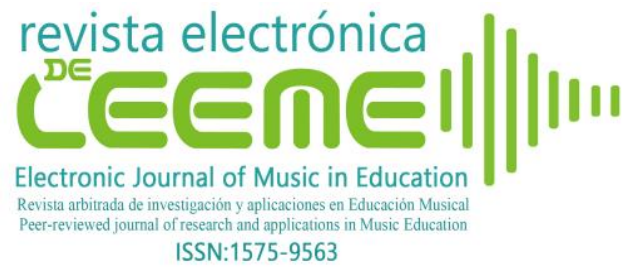

\begin{tabular}{|c|c|c|c|c|}
\hline & Currículo de Educación Musical & $1^{\circ}$ & 2 & OP \\
\hline & Didáctica Especial de Música & $1^{\circ}$ & 2 & OP \\
\hline & Practicum I & $1^{\mathrm{o}}$ & 2 & $\mathrm{PE}$ \\
\hline Universidad & Practicum II & $1^{\circ}$ & 2 & $\mathrm{PE}$ \\
\hline Pontificia de & Practicum III & $1^{\circ}$ & 2 & $\mathrm{PE}$ \\
\hline $\begin{array}{c}\text { Salamanca } \\
\text { (UPSA) }\end{array}$ & TFM & $1^{\mathrm{o}}$ & no especificado & TFM \\
\hline
\end{tabular}

Fuente: elaboración propia a partir de los datos obtenidos

La recogida de los datos se llevó a cabo utilizando una rúbrica diseñada en el marco del Proyecto Profmus y basada en las conclusiones del proyecto ALFA (Aróstegui, 2010), que consta de un apartado general sobre el plan de estudios y otro con aspectos concretos para cada una de las asignaturas (Tabla 3).

Tabla 3. Elementos de análisis de la rúbrica para cada plan de estudios y asignatura del plan de estudios

Aspectos generales de cada plan

\section{Perfil de ingreso}

Perfil de egreso

Procedimiento de evaluación del título

Seriación y articulación de las asignaturas

Modelos educativos

Pedagogías musicales de referencia Énfasis del plan en la formación de profesorado
Aspectos de cada asignatura

\author{
Objetivos \\ Contenidos \\ Evaluación
}

Métodos de enseñanza

Bibliografía

Observaciones de cada asignatura Áreas que la imparten

Fuente: elaboración propia sobre rúbrica del proyecto Profmus

El análisis de los datos se llevó a cabo a través de codificación axial (Strauss y Corbin, 1990) categorizando línea a línea toda la documentación volcada en la rúbrica, utilizando el software ATLAS.ti (versión 8.1). Los códigos se agruparon en categorías de orden superior y, en paralelo, se llevó a cabo una categorización del currículo actual de las Enseñanzas Obligatorias. Un siguiente nivel de trabajo consistió en encontrar códigos de intersección entre la formación universitaria y las directrices marcadas por la legislación, que resultaron ser ocho (Tabla 4). 
Tabla 4. Códigos de intersección entre planes de estudios y legislación en Primaria y Secundaria

\begin{tabular}{|c|c|c|c|c|}
\hline Dimensiones & $\begin{array}{l}\text { Subcategorías de } \\
\text { intersección }\end{array}$ & $\begin{array}{l}\text { Documentación } \\
\text { analizada: } \\
\text { Primaria y } \\
\text { Secundaria }\end{array}$ & $\begin{array}{l}\text { Documentación } \\
\text { analizada: } \\
\text { Educación } \\
\text { Superior }\end{array}$ & Descripción \\
\hline \multirow{4}{*}{$\begin{array}{l}\text { Contenidos } \\
\text { musicales }\end{array}$} & $\begin{array}{c}\text { Escucha } \\
\text { Interpretación } \\
\text { musical y creación }\end{array}$ & \multirow{2}{*}{$\begin{array}{l}\text { Bloques de } \\
\text { contenidos de } \\
\text { las asignaturas } \\
\text { de Primaria. } \\
\text { Currículo }\end{array}$} & \multirow{2}{*}{$\begin{array}{l}\text { Contenidos } \\
\text { asignaturas } \\
\text { Mención. Memoria } \\
\text { de verificación y } \\
\text { guías docentes }\end{array}$} & \multirow{2}{*}{$\begin{array}{l}\text { Analiza cómo se abordan y } \\
\text { correlacionan la escucha; } \\
\text { interpretación musical y } \\
\text { creación; danza y movimiento } \\
\text { en los currículos de Ed. } \\
\text { Primaria y del Grado } \\
\text { (Mención) }\end{array}$} \\
\hline & $\begin{array}{c}\text { Danza y } \\
\text { movimiento }\end{array}$ & & & \\
\hline & $\begin{array}{c}\text { Contextos musicales } \\
\text { y Culturales }\end{array}$ & \multirow{2}{*}{$\begin{array}{l}\text { Asignaturas de } \\
\text { Educación } \\
\text { Secundaria. } \\
\text { Currículo }\end{array}$} & \multirow{2}{*}{$\begin{array}{l}\text { Contenidos } \\
\text { asignaturas del } \\
\text { Máster. Memoria } \\
\text { de verificación y } \\
\text { guías docentes }\end{array}$} & \multirow{2}{*}{$\begin{array}{l}\text { Analiza cómo se abordan y } \\
\text { correlacionan: interpretación y } \\
\text { creación; escucha; contextos } \\
\text { musicales y culturales; música } \\
\text { y tecnologías en ESO y } \\
\text { Máster de Profesorado }\end{array}$} \\
\hline & $\begin{array}{l}\text { Música y } \\
\text { tecnologías }\end{array}$ & & & \\
\hline \multirow{3}{*}{$\begin{array}{l}\text { Contenidos } \\
\text { transversales }\end{array}$} & $\begin{array}{l}\text { Comprensión lectora } \\
\text { y expresión oral y } \\
\text { escrita }\end{array}$ & \multirow{3}{*}{$\begin{array}{l}\text { Contenidos } \\
\text { transversales. } \\
\text { Currículo }\end{array}$} & $\begin{array}{l}\text { Métodos docentes y } \\
\text { evaluación. Guías } \\
\text { docentes }\end{array}$ & $\begin{array}{l}\text { Utilización de la lengua para } \\
\text { la comprensión de textos y la } \\
\text { producción de estos, tanto } \\
\text { escritos como orales }\end{array}$ \\
\hline & Emprendimiento & & $\begin{array}{c}\text { Pedagogías } \\
\text { musicales de } \\
\text { referencia, } \\
\text { bibliografía, } \\
\text { evaluación. Guías } \\
\text { docentes }\end{array}$ & $\begin{array}{l}\text { Reconocimiento de la música } \\
\text { como medio de expresión y } \\
\text { estímulo de la creatividad, el } \\
\text { trabajo individual y en equipo, } \\
\text { la autonomía, el espíritu } \\
\text { emprendedor }\end{array}$ \\
\hline & $\begin{array}{l}\text { Ciudadanía y } \\
\text { diversidad }\end{array}$ & & $\begin{array}{c}\text { Objetivos, } \\
\text { contenidos, } \\
\text { métodos docentes, } \\
\text { bibliografía. } \\
\text { Memoria } \\
\text { verificación y guías } \\
\text { docentes }\end{array}$ & $\begin{array}{l}\text { Reconocimiento y actuación } \\
\text { sobre la diversidad en el aula, } \\
\text { la diversidad cultural, género, } \\
\text { y ciudadanía }\end{array}$ \\
\hline
\end{tabular}

Fuente: elaboración propia

\section{Resultados}

A continuación, se presentan los resultados agrupados por cada una de las categorías de intersección emergentes del análisis cualitativo. 


\subsubsection{Los contenidos de Música en los currículos de Educación Primaria y de Educación Secundaria Obligatoria (ESO)}

Esta dimensión analiza en qué medida los diversos bloques de los currículos de Ed. Primaria y de ESO (España. Ministerio de Educación, 2014) están representados en las asignaturas de los planes de estudio universitarios. Se ha estudiado si existen asignaturas específicas que aborden dichos contenidos y, si no es así, se ha analizado en qué medida dichos contenidos se incluyen en otras asignaturas.

El análisis de los contenidos del Grado de Maestro/a y del Máster muestra que no en todos los casos se contempla una asignatura específica para cada bloque de estándares de aprendizaje previstos en la legislación. Se analizan a continuación la escucha; la interpretación musical; la música, el movimiento y la danza; contextos musicales y culturales; y música y tecnologías, como bloques curriculares recogidos en la Enseñanza Obligatoria.

\subsubsection{Escucha}

El currículum de Educación Primaria plantea para este bloque la escucha y las propiedades del sonido como punto de partida para realizar creaciones propias, el análisis de obras musicales sencillas y la valoración del patrimonio musical.

En cuanto a la formación de docentes, ULE, UBU y UPSA incluyen asignaturas específicas o compartidas de educación auditiva. En ellas se abordan aspectos como el paisaje sonoro, el sonido y sus cualidades, ruido, silencio, los procesos cognitivos implicados en la audición, la memoria musical o la pedagogía de la audición. Otras universidades no contemplan asignaturas específicas sobre educación auditiva, aunque incluyen estos contenidos en asignaturas genéricas de didáctica de la música para maestros generalistas.

En la ESO, la escucha aborda la discriminación auditiva, la valoración del silencio, las músicas de diversas épocas y culturas, sus elementos compositivos y la contaminación acústica, entre otros aspectos. Sin embargo, en los planes de estudio de profesorado apenas se reflejan contenidos relacionados con la escucha, sólo se identifican en Didáctica de la Música (UVa y USAL). Si bien el alumnado que cursa el Máster tiene formación musical previa, sin duda, la parte de didáctica de la escucha se aborda escasamente.

\subsubsection{Interpretación musical}

El currículo de Educación Primaria recoge para este bloque aspectos relacionados con el uso de la voz como instrumento expresivo y sus posibilidades para interpretar, crear o 


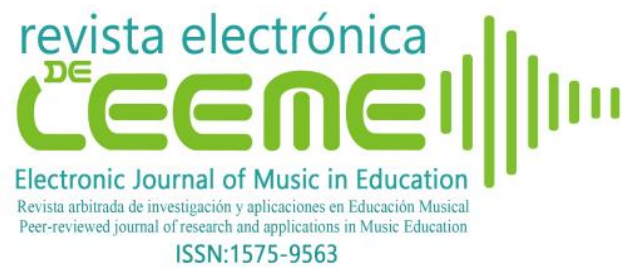

improvisar. También la interpretación instrumental individual y grupal y la exploración de las posibilidades sonoras de diversos materiales y dispositivos electrónicos.

Todos los planes de estudio del Grado de Educación Primaria contienen asignaturas específicas sobre interpretación musical. Algunas están separadas en formación vocal o instrumental, y en otros se contemplan de forma conjunta. Los contenidos que abordan son muy diversos, desde la interpretación con flauta dulce, instrumentos escolares, la elaboración de arreglos, el acompañamiento, hasta la interpretación y dirección corales. Llama la atención, sin embargo, que la creación no ocupe un lugar destacado dada la necesidad propuesta en el currículo.

Por otra parte, tampoco se encuentran suficientes referencias a la exploración de materiales diversos (además de instrumentos), salvo en UVa y ULE, donde incluyen la exploración con cuerpos sonoros. Los instrumentos digitales, sin embargo, están presentes en mayor o menor medida en todos los planes de estudio analizados.

En la ESO, la interpretación musical y la creación aparecen unidas en un mismo bloque de la legislación. Además de los contenidos de lenguaje musical se plantea la exploración de distintas fuentes y objetos y se hace referencia expresa a la creación de paisajes sonoros. En cuanto al Máster, ambos procesos aparecen reflejados en la asignatura Didáctica de la Música (UVa y USAL).

\subsubsection{La música, el movimiento y la danza}

El currículo de Educación Primaria tiene previsto en este bloque la adquisición de capacidades expresivas y creativas que ofrecen la expresión corporal y la danza, valorando su aportación al patrimonio y disfrutando de su interpretación como una forma de interacción social.

Diversas universidades contienen en su plan de estudios contenidos relacionados con la danza. No obstante, la expresión corporal aparece vinculada al área de Educación Física. La UVa, USAL y ULE incluyen entre sus asignaturas Formación Rítmica y Danza. De alguna manera, al agruparlas en una misma asignatura, se concede importancia a la formación rítmica a través del cuerpo, un aspecto que diversos métodos activos tienen en cuenta. En el resto de las universidades, la danza forma parte de los contenidos de otras asignaturas del currículo, tanto las que son comunes a los maestros generalistas como de la Mención.

En la ESO, la danza forma parte de las asignaturas optativas, aunque, a diferencia de la música, sólo se oferta en el $4^{\circ}$ curso y aparece vinculada con las Artes Escénicas. Entre los estándares de aprendizaje, destacan la interpretación de un repertorio de danzas en grupo y el

@Yurima Blanco García y Alicia Peñalba Acitores. The content of this article is the sole responsibility of the authors. The Revista Electrónica de LEEME and Universitat de València are not liable for any legal actions that may arise involving the article's content. Revista Electrónica de LEEME - Lista Electrónica Europea de Música en la Educación-. http://ojs.uv.es/index/php/LEEME/index ISSN: 1575-9563. Editores: Universidad de Valencia y Jesús Tejada. Visibilidad de esta revista: SCOPUS, Emerging Sources Citation Index (Clarivate), EBSCO, CINDOC (CSIC), Citefactor, COPAC, Dialnet, DICE (CSIC), DOAJ, e-revistas (CSIC), EBSCO Premier, ERIH+, Gale Cengage Learning, IN-RECS, IRESIE, LATINDEX, MIAR, OCLC Worldcat, RESH, REDIB, RILM Core Journals, SUDOC, ULRICHS. Esta revista es de acceso libre mediante licencia Creative Commons 4.0 CC by. Política de archivado: etiqueta verde SHERPA-ROMEO. 


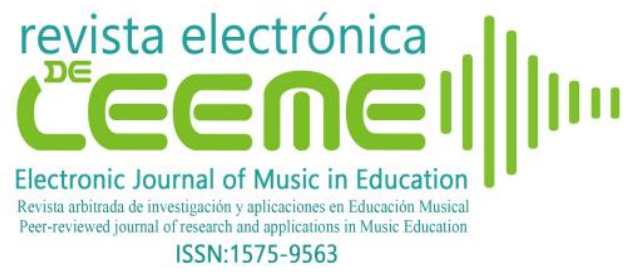

uso preciso del movimiento en la improvisación. Paralelamente, en la asignatura de música se incluyen aspectos vinculados con la danza, por ejemplo, el reconocimiento e interpretación de repertorios tradicionales. En cuanto al Máster, la danza aparece reflejada muy sucintamente en la asignatura Didáctica de la Música (UVa y USAL) y Metodología en la especialidad de Música (USAL).

\subsubsection{Contextos musicales y culturales}

Si bien no se refleja este tema como un bloque específico en Primaria, se constata el interés por abarcar los contextos musicales y culturales de manera transversal en otros apartados, como en patrimonio, valores sociales y cívicos y educación artística. Algunas asignaturas del Grado incorporan este tema en los contenidos (Formación Instrumental, UBU); objetivos (Formación Vocal y Auditiva, UPSA; Lenguaje Musical, ULE) o dedican una asignatura específica a ello (Música, Cultura y Diversidad, UVa). Otras reflejan el folklore infantil en sus guías, pero no especifican si incluyen el de Castilla y León.

En la ESO, Contextos Musicales y Culturales comprende uno de los bloques de contenidos y sus objetivos se orientan hacia el reconocimiento de la música en diversos contextos culturales. Se enfatiza en el conocimiento del folklore y la tradición cultural de España, así como de otras regiones geográficas, a la vez que proponen diversificar los referentes culturales de los educandos. En cuanto al Máster, aparece reflejado en los contenidos de Teoría de la Música y Prácticas Musicales Tradicionales y Populares (UVa).

\subsubsection{Música y tecnologías}

La competencia digital constituye una de las habilidades transversales propuestas por la legislación, de ahí que aparezca comprendida en todas las áreas de Educación Primaria y ESO. Los contenidos se orientan hacia una doble perspectiva: la adquisición de habilidades técnicas y de funcionamiento de herramientas audiovisuales digitales; y el desarrollo de aspectos creativos, colaborativos y de pensamiento crítico.

En Educación Primaria, aparece vinculada al bloque de interpretación (sonorización, grabación, uso de lenguaje audiovisual). En cuanto al Grado, algunos programas contienen una asignatura específica sobre TICs o incluyen el uso de las tecnologías con el objetivo de grabar, editar o diseñar actividades con soporte informático. Todas coinciden en la necesidad de utilizar las TICs de forma crítica y responsable. La UVa incluye dos asignaturas: TICs Aplicadas a la Educación Musical, centrada en aplicar didácticamente los medios audiovisuales e informáticos como recursos para componer, elaborar arreglos, editar partituras y sonido y crear producciones 


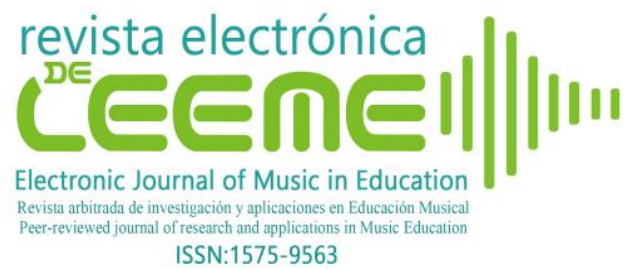

multimedia; y Creación Artística, Cultura Visual y Musical, orientada a la elaboración y análisis de soportes visuales y audiovisuales.

Música y Tecnologías constituye uno de los bloques de contenidos en la ESO. Se prevé la aplicación de herramientas tecnológicas para la actividad musical y, de manera específica, el desarrollo de creaciones sonoras y audiovisuales. En el Máster, las TICs se reflejan en la mayoría de las asignaturas como una de las competencias transversales que debe alcanzar el alumnado, sin embargo, los contenidos sólo se concretan en dos asignaturas: Recursos en la Especialidad de Música (USAL) y Teoría de la Música y Prácticas Musicales Tradicionales y Populares (UVa).

\subsection{Contenidos transversales y objetivos de la educación}

Además de los contenidos musicales del currículo, antes mostrados mediante el análisis de las asignaturas universitarias y de la Enseñanza Obligatoria, es importante estudiar otros aspectos curriculares transversales que vertebran todos los aprendizajes y relacionarlos con los objetivos que plantea la legislación. El propósito es comprender en qué medida el futuro profesorado recibe formación suficiente en este sentido. Las categorías relevantes que emergen del análisis de los datos se relacionan con tres pilares: 1) expresión oral y escrita; 2) emprendimiento y creatividad; y, por último, 3) valores cívicos y respeto a la diversidad.

\subsubsection{Expresión oral y escrita}

Aunque los estudiantes en educación superior reciben diversas asignaturas relacionadas con la expresión oral y escrita y el uso de la lengua, no es desdeñable analizar en qué medida en las asignaturas de música se da también un valor a esta competencia transversal. Se confirma que gran parte de las asignaturas de todas las universidades incluyen la comprensión y lectura de textos, así como la presentación de forma oral o escrita de diversos trabajos, quizá con mayor presencia que en otras áreas, en las que la evaluación está más orientada hacia la realización de exámenes finales. En el caso de las asignaturas de Música, la mayor parte incluyen la evaluación continua.

\subsubsection{Emprendimiento y creatividad}

La autonomía, el emprendimiento y la creatividad se perfilan como competencias transversales en las etapas de Primaria y Secundaria. La creación musical en Primaria aparece fusionada en los contenidos de interpretación, mientras que en la ESO ambos procesos conforman un bloque específico. Para su revisión en los planes de estudio universitarios se ha

@Yurima Blanco García y Alicia Peñalba Acitores. The content of this article is the sole responsibility of the authors. The Revista Electrónica de LEEME and Universitat de València are not liable for any legal actions that may arise involving the article's content. Revista Electrónica de LEEME - Lista Electrónica Europea de Música en la Educación-. http://ojs.uv.es/index/php/LEEME/index ISSN: 1575-9563. Editores: Universidad de Valencia y Jesús Tejada. Visibilidad de esta revista: SCOPUS, Emerging Sources Citation Index (Clarivate), EBSCO, CINDOC (CSIC), Citefactor, COPAC, Dialnet, DICE (CSIC), DOAJ, e-revistas (CSIC), EBSCO Premier, ERIH+, Gale Cengage Learning, IN-RECS, IRESIE, LATINDEX, MIAR, OCLC Worldcat, RESH, REDIB, RILM Core Journals, SUDOC, ULRICHS. Esta revista es de acceso libre mediante licencia Creative Commons 4.0 CC by. Política de archivado: etiqueta verde SHERPA-ROMEO. 


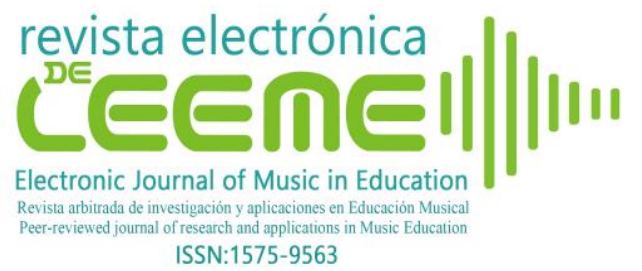

llevado a cabo un análisis de los contenidos, métodos de enseñanza, evaluación, modelos educativos y bibliografía recogidos en las guías docentes de Grado y Máster.

En el Grado, solamente la UBU y la USAL incluyen asignaturas específicas relacionadas con la creación: Composición y Creación Musical y Creación y Selección de Repertorio Musical para el Aula, respectivamente. La primera aborda la armonía básica, la improvisación en un instrumento melódico-armónico y la creación musical a través de textos, otorgándole un papel protagonista como vertiente natural de la enseñanza y la práctica musical en el aula. La segunda plantea la capacidad para crear acompañamientos y arreglos, adaptar repertorios y adquirir destrezas para expresarse musicalmente. Aunque no conciban la creación musical como asignatura, otras universidades contemplan también esta competencia: la ULE incluye la creación de coreografías y recursos y la UPSA, la creación de canciones. En los planes de Máster, no se especifican contenidos de creación musical, no obstante, aparecen reflejados como objetivos de la asignatura Didáctica de la Música (UVa y USAL).

Respecto a los métodos de enseñanza, la revisión de las guías de Grado y Máster permite reconstruir, en cierta medida, el tipo de pedagogías de referencia utilizadas. En las asignaturas de Mención, se trabaja fundamentalmente los métodos activos (Dalcroze, Orff,), aunque en algunas universidades, como la USAL, se abordan también pedagogías creativas basadas en Delalande, Dennis, Paynter y Schaffer.

Muchas de las asignaturas utilizan metodologías que favorecen la autonomía y el emprendimiento. Se promueve la asistencia a conciertos, el aprendizaje a través de la experiencia musical, la participación en el coro universitario (UBU), la exposición de materiales teórico-prácticos en clase, el aprendizaje basado en proyectos, la creación de canciones y arreglos musicales para su interpretación en el aula, entre otras estrategias que fomentan la iniciativa personal y colectiva. Asimismo, en las tareas de evaluación de las asignaturas de música, a diferencia de otras materias, se fomenta el trabajo colaborativo grupal. Aunque el trabajo individual está presente en todas las universidades y prácticamente en todas las asignaturas, el trabajo grupal constituye uno de los pilares de la evaluación. En este sentido, se pretende desarrollar y mejorar la convivencia grupal, el respeto de las ideas propias y colectivas, las habilidades sociales y la cooperación.

\subsubsection{Ciudadanía y diversidad}

Uno de los elementos destacados que aborda la legislación, tanto en sus competencias transversales como en sus objetivos, son los valores cívicos y ciudadanos y el respeto a la diversidad. Son heterogéneas las referencias que muestran los planes de estudio respecto a la diversidad en el aula. En su mayor parte aluden a la capacidad del docente de implementar actuaciones para el alumnado con diversidad funcional. No obstante, la diversidad también está

@Yurima Blanco García y Alicia Peñalba Acitores. The content of this article is the sole responsibility of the authors. The Revista Electrónica de LEEME and Universitat de València are not liable for any legal actions that may arise involving the article's content. Revista Electrónica de LEEME - Lista Electrónica Europea de Música en la Educación-. http://ojs.uv.es/index/php/LEEME/index ISSN: 1575-9563. Editores: Universidad de Valencia y Jesús Tejada. Visibilidad de esta revista: SCOPUS, Emerging Sources Citation Index (Clarivate), EBSCO, CINDOC (CSIC), Citefactor, COPAC, Dialnet, DICE (CSIC), DOAJ, e-revistas (CSIC), EBSCO Premier, ERIH+, Gale Cengage Learning, IN-RECS, IRESIE, LATINDEX, MIAR, OCLC Worldcat, RESH, REDIB, RILM Core Journals, SUDOC, ULRICHS. Esta revista es de acceso libre mediante licencia Creative Commons 4.0 CC by. Política de archivado: etiqueta verde SHERPA-ROMEO. 


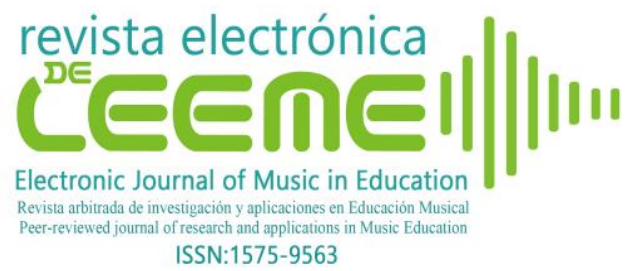

presente en la selección de repertorio musical de otras culturas y estilos diferentes a la denominada música culta occidental, en la valoración del patrimonio propio y el conocimiento de los valores ciudadanos de la música.

El análisis de las guías de Grado y Máster pone de manifiesto una ausencia de concepciones referentes al género, tanto en los contenidos como en los objetivos de las asignaturas, excepto en los programas de la UVa y la USAL, algo que no se materializa después en los contenidos. Destaca en el Grado la asignatura Música, Cultura y Diversidad (UVa), que pretende utilizar la música como elemento integrador, fomentar prácticas de relación intercultural, inclusión social, intergeneracionales y de igualdad, además de seleccionar un repertorio equilibrado y diverso, planificar actividades que fomenten el respeto hacia las diferentes manifestaciones culturales y el uso de la música como herramienta para mejorar la calidad de vida y la salud. Por su parte, Música y Danza para la Diversidad (USAL) aborda, entre otras cuestiones, la diversidad y su atención educativa desde el área artística.

En la siguiente imagen (Figura 1), se muestra los principales resultados de la investigación. De cada una de las ocho categorías analizadas, se comenta (en color verde) su característica en los diversos planes de estudio. Además, en el extremo final del esquema, se incluyen las universidades que destacan por contemplar los contenidos en mayor medida.

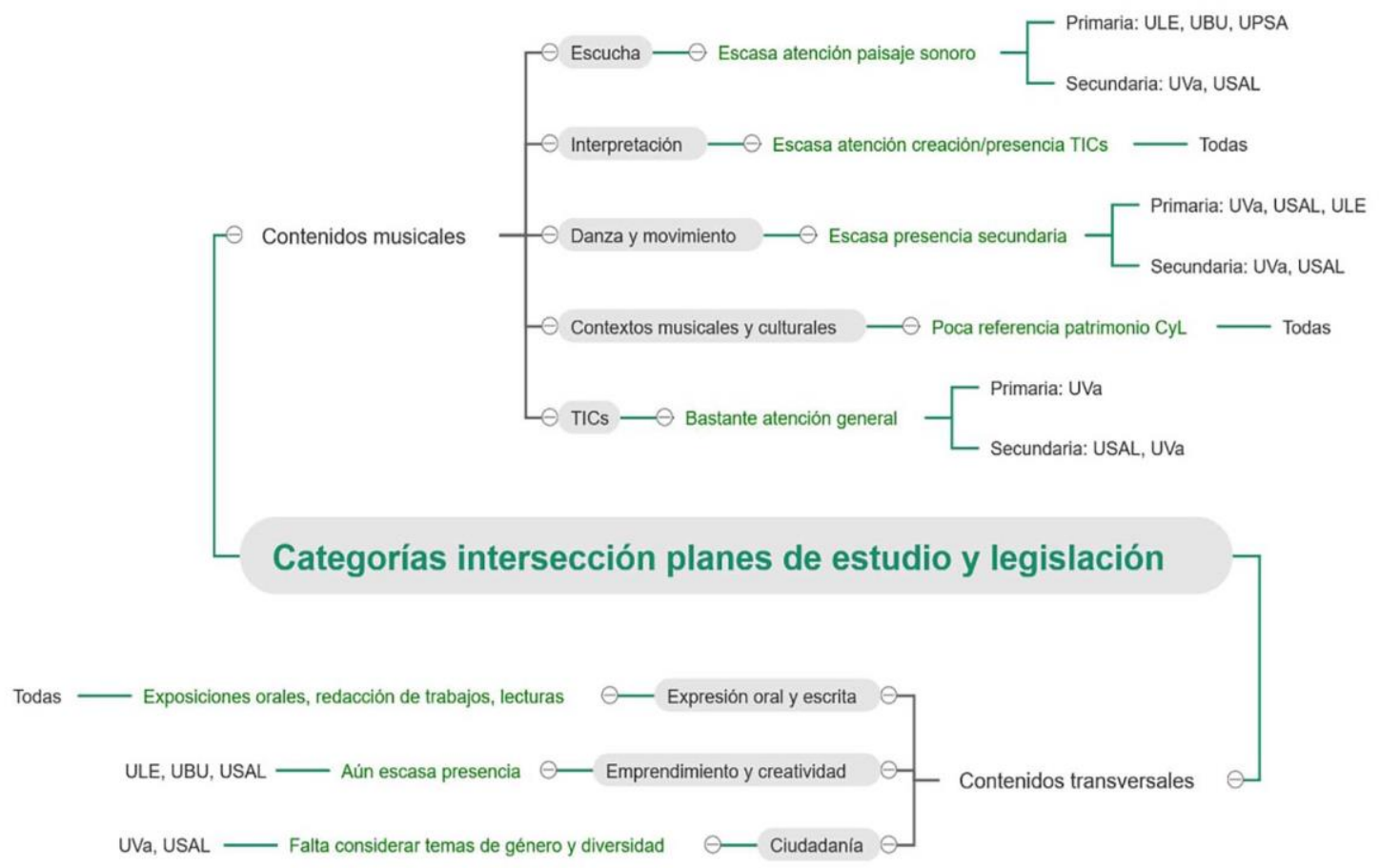

Figura 1. Resultados de intersección entre planes de estudio y legislación. Fuente: elaboración propia 


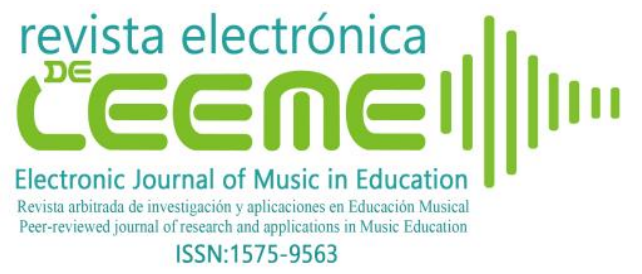

\section{Discusión y conclusiones}

El análisis de los planes de estudios examinados pone de manifiesto la tendencia general que se observa en el país. La formación de maestros con mención de música se ha visto reducida con respecto a los estudios de diplomatura (Domínguez, 2014) y no resulta suficiente (RosaNapal et al., 2020). La mención consta, aproximadamente, de cinco asignaturas de especialidad. El alumnado recibe una formación didáctica mucho más completa, pero la formación musical, reducida a cerca de 30 ECTS, es limitada. En la mayor parte de las universidades, no se exige un perfil de ingreso determinado, por lo que muchos estudiantes que acceden a la mención no tienen conocimientos musicales previos (López-Reyes, 2010). Es necesario valorar esta formación de manera prospectiva, con la mirada puesta en el docente de música del futuro y no sobre necesidades de corto plazo (Aróstegui y Kyakuwa, 2020).

Por su parte, el Máster de Secundaria ha enriquecido la formación del alumnado respecto al antiguo Certificado de Actitud Pedagógica (CAP). En dos de las tres universidades estudiadas - UVa y USAL-, consta de dos cursos muy intensivos, con periodo de prácticas y un trabajo de fin de estudios que contribuyen a la calidad de la formación. Las asignaturas incluyen innovación e investigación musicales, además de un perfil pedagógico, pues no olvidemos que este alumnado carece de formación didáctica.

Como se expuso en los resultados, el examen señala algunos puntos débiles en la correlación entre el plan de estudios y las competencias que establece el currículo de enseñanza obligatoria.

Respecto a los contenidos musicales de Grado, no se hace suficiente hincapié en el desarrollo de la escucha para la creación (Carrillo y Vilar, 2014). También resulta insuficiente en el Máster, teniendo en cuenta que la escucha ocupa uno de los bloques del $1^{\circ}$ y $4^{\circ}$ curso de ESO. La interpretación musical aparece recogida en todos los planes y muestra diversidad de enfoques y prácticas vocales e instrumentales, sin embargo, la exploración con cuerpos sonoros y timbres y, por tanto, un mayor acercamiento a la realidad sonora contemporánea aparece sutilmente solo en algunos planes. Por último, la presencia minimizada de la danza y la expresión corporal no parece coherente con su valor en el currículo de la ESO.

Por otra parte, tanto el currículo de Primaria como el de ESO hacen hincapié en la necesidad de conocer, valorar y cuidar el patrimonio musical propio. Sin embargo, se observan escasas referencias y recursos para trabajar el patrimonio regional, lo que parece obviar su importancia como elemento clave de la educación musical (Chao-Fernández, Gisbert y ChaoFernández, 2020). A diferencia de otras comunidades autónomas en las que el trabajo patrimonial tiene un gran peso (Riaño y Cabedo-Mas, 2013), en nuestros planes suele estar presente en el repertorio o como temática de algunas materias, pero no se dedican programas a su estudio, práctica y formación didáctico-artística. Por último, en cuanto al binomio música y 


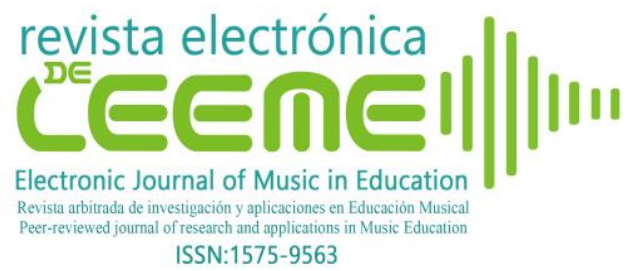

tecnologías, se referencia ampliamente en la formación del profesorado, ya sea como asignatura específica o enfocada como una competencia transversal (Calderón et al., 2020).

Las asignaturas del plan de estudios, en general, se adaptan a las necesidades profesionales de los docentes en la actualidad, aunque existen algunos aspectos que podrían replantearse respecto a las nuevas realidades educativas y sociales. Se identifican necesidades relacionadas con la formación a lo largo de la vida (Ballantyne, 2007) y la concreción de un perfil crítico, reflexivo y creativo (Elliott y Silverman, 2019) en la formación del profesorado de música de Castilla y León.

Uno de los retos en la formación de docentes es la escasez de tiempo y de asignaturas específicas para una sólida especialización. En este sentido, los maestros y profesores suelen exhortar el aprendizaje a lo largo de la vida de sus estudiantes. Las actividades extraescolares, los coros, el autoaprendizaje a través de canales de YouTube, son algunas de las estrategias de las que se sirve el profesorado para alentar este tipo de actitudes (Esteve-Faubel, Molina y Esteve-Fauvel, 2009). La educación musical a lo largo de la vida es sin duda un elemento clave para el éxito en una sociedad como la nuestra, en la que la infancia participa poco de la actividades musicales (Small, 2003). Ello podría ser una clave para el diseño de planes de estudios que adolecen de escasos, pero que pueden complementarse con otras actividades musicales universitarias: coros, batucadas, danza, conjuntos instrumentales, bandas y otros semejantes. Asimismo, fomentar el aprendizaje fuera del aula permite también el desarrollo de una identidad universitaria, de pertenencia a la institución, trabajo en equipo y acción comunitaria.

La educación musical debe contribuir a la formación de ciudadanos críticos, emprendedores y creativos (Peñalba, 2017), dispuestos a afrontar los retos de la sociedad actual. Un primer eslabón recae en el docente de música, de ahí la necesidad de plantear una formación integral, flexible y adecuada a la realidad a la par que creativa y emprendedora. Los planes de estudio universitarios atienden estos aspectos en los contenidos transversales del currículo. Sin embargo, no parece suficiente abordar estos ejes competenciales sin una adecuada sinergia entre ellos en relación al contexto de formación y actuación de los futuros docentes. Tomar en cuenta la realidad sociocultural de Castilla y León será crucial para articular estrategias vinculadas con el patrimonio, el respeto a la diversidad cultural y de género, el emprendimiento, la sostenibilidad, el desarrollo tecnológico, entre otras áreas clave para una participación de la música y la educación en el desarrollo humano y social.

\section{Financiación y agradecimientos}

Este trabajo ha sido financiado por el Ministerio de Industria, Economía y Competitividad, en su Programa Estatal de Fomento de la Investigación Científica y Técnica de Excelencia (Proyecto I+D 
código EDU2017-84782, "Formación de profesorado y música en la sociedad y la economía del conocimiento", Profmus).

\section{Referencias}

Aróstegui, J.L. (Ed.) (en prensa). La educación musical escolar y las demandas del sistema escolar: del arte por amor al arte a la Sociedad y la Economía del Conocimiento. Barcelona: Octaedro.

Aróstegui, J.L. (2006). La formación del profesorado en Educación Musical ante la Convergencia Europea en Enseñanzas Universitarias. Revista de Educación, 341(septiembre-diciembre), 829-844. Recuperado de: http://www.educacionyfp.gob.es/dam/jcr

Aróstegui, J.L. (2010). Formación del profesorado de música: planes de estudio en Europa y América Latina. Profesorado. Revista de Currículum y Formación del Profesorado, 14(2), 3-7. Recuperado de: https://recyt.fecyt.es/index.php/profesorado/

Aróstegui, J.L. y Cisneros-Cohernour, E. (2010). Reflexiones en torno a la formación del profesorado de música a partir del análisis documental de los planes de estudio en Europa y América Latina. Profesorado. Revista de Currículum y Formación del Profesorado, 14(2), 179-189. Recuperado de: https://recyt.fecyt.es/index.php/profesorado/

Aróstegui, J.L., Louro, A.L. y Teixeira, Z.L. (2015). Las políticas educativas de reforma y su impacto en la Educación Musical Escolar. De dónde venimos y hacia dónde podemos ir. Revista da ABEM, 23(35), 24-34. Recuperado de: http://abemeducacaomusical.com.br/revistas/revistaabem/index.php/revistaabem/article/

Aróstegui, J.L. y Kyakuwa, J. (2020). Generalist or specialist music teachers? Lessons from two continents. Arts Education Policy Review. doi:10.1080/10632913.2020.1746715

Ballantyne, J. (2007). Integration, contextualization and continuity: three themes for the development of effective music teacher education programmes. International Journal of Music Education, 25(2), 119-136. doi:10.1177/0255761407079955

Cabedo-Mas, A. (2011). La educación musical a lo largo de la vida. En Actas del IV Congreso Iberoamericano de Universidades para Mayores, CIUUM 2011, "Aprendizaje a lo largo de la vida, envejecimiento activo y cooperación internacional en los programas universitarios para mayores" (Alicante, del 27 al 30 de junio de 2011) (pp.191-208). Recuperado de: http://rua.ua.es/dspace/handle/10045/20104

Calderón, D., Gustems, J. y Carrera, X. (2020). Música y TIC en los grados de maestro:

@Yurima Blanco García y Alicia Peñalba Acitores. The content of this article is the sole responsibility of the authors. The Revista Electrónica de LEEME and Universitat de València are not liable for any legal actions that may arise involving the article's content. Revista Electrónica de LEEME - Lista Electrónica Europea de Música en la Educación-. http://ojs.uv.es/index/php/LEEME/index ISSN: 1575-9563. Editores: Universidad de Valencia y Jesús Tejada. Visibilidad de esta revista: SCOPUS, Emerging Sources Citation Index (Clarivate), EBSCO, CINDOC (CSIC), Citefactor, COPAC, Dialnet, DICE (CSIC), DOAJ, e-revistas (CSIC), EBSCO Premier, ERIH+, Gale Cengage Learning, IN-RECS, IRESIE, LATINDEX, MIAR, OCLC Worldcat, RESH, REDIB, RILM Core Journals, SUDOC, ULRICHS. Esta revista es de acceso libre mediante licencia Creative Commons 4.0 CC by. Política de archivado: etiqueta verde SHERPA-ROMEO. 
contenidos y actividades en los planes docentes. En Llibre d'actes de la I Conferència Internacional de Recerca en Educació. Educació 2019: reptes, tendències $i$ compromisos (4 i 5 de novembre de 2019, Universitat de Barcelona) (pp.256-262). Barcelona: Institut de Recerca en Educació (IRE-UB). Recuperado de: http://hdl.handle.net/2445/164677

Carrillo, C. y Vilar, M. (2014). El perfil profesional del profesorado de música: una propuesta de las competencias deseables en Ed. Primaria y Ed. Secundaria. Revista Electrónica de LEEME, 33, 1-26. Recuperado de: https://ojs.uv.es/index.php/LEEME/article/view/9856/9276

Conway, C., Pellegrino, K., Stanley, A. M. y West, Ch. (2019). The Oxford Handbook of Preservice Music Teacher Education in the United States. New York: Oxford University Press. doi:10.1093/oxfordhb/9780190671402.001.0001

Chao-Fernández, R., Gisbert, V. y Chao-Fernández, A. (2020). Contribución a la conservación del patrimonio musical en Educación Primaria. Estudio de caso en Galicia en 2003 y 2019. Revista Electrónica de LEEME, 45, 111-125. doi:10.7203/LEEME.45.16880

Chiva, O., Salvador, C., Ferrando, S. y Cabedo-Mas, A. (2019). Service learning in musical education: Literature review and recommendations for practice. Revista Electrónica Complutense de Investigacion en Educacion Musical, 16, 57-74. doi:10.5209/reciem.62409

Domínguez, M.B. (2014). La Educación Musical en el desarrollo de la LOMCE en Aragón. Fórum Aragón, 12, 50-52. Recuperado de: https://dialnet.unirioja.es/servlet/articulo?coridgo $=4754435$

Elliott, D.J. y Silverman, M. (2019). Change in Music Teacher Education. En C. Conway, C. Pellegrino, K. Stanley y A. West (Eds.), The Oxford Handbook of Preservice Music Teacher Education in the United States (sin paginar). New York: Oxford University Press. doi:10.1093/oxfordhb/9780190671402.013.4

Esteve-Faubel, J.M., Molina, M.A. y Esteve-Fauvel, R.P. (2009). Autoaprendizaje en el EEEs. una experiencia en Magisterio Especialidad Musical. Revista de Investigación Educativa, 27(2), 337-351. Recuperado de: https://revistas.um.es/rie/article/view/94441

Futured (27 de mayo de 2020). Music Teacher Education for the Future (Futured). Recuperado de: https://prosjekt.hvl.no/futured/

Gillanders, C., Torres, A.C. y Pérez, L.T. (2018). Music education and service-learning: A case study in Primary Education teacher training. Revista Electrónica de LEEME, 2(42), 16- 


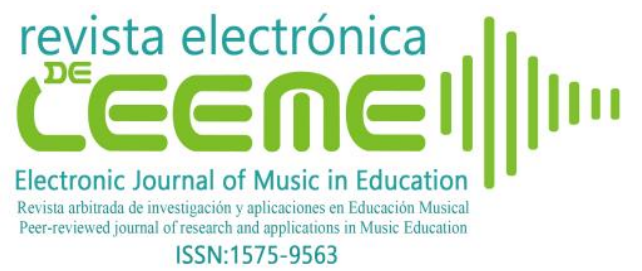

30. doi:10.7203/LEEME.42.12329

Ivanova, A. y Siebenaler, D. (2018). La formación del profesorado de música en la Universidad Complutense de Madrid y la Universidad Estatal de California: Un estudio comparado. Profesorado. Revista de Currículum y Formación del Profesorado, 22, 295-315. doi:10.30827/profesorado.v22i3.8003

López-García, N.J., Madrid, D. y De Moya, M. V. (2017). La formación musical en los planes de estudios para maestros de Primaria en la Universidad de Castilla-La Mancha. Estudios Pedagógicos, 43(1), 423-438. doi:10.4067/S0718-07052017000100024

Mateos-Moreno, D. (2013). Perfil del profesorado de música en Educación Primaria durante su etapa de formación universitaria. Revista Electrónica de LEEME, 31, 52-71. Recuperado de: https://ojs.uv.es/index.php/LEEME/article/view/9847/9268

Ministerio de Educación y Ciencia (2007a). Orden ECI/3857/2007, de 27 de diciembre, por la que se establecen los requisitos para la verificación de los títulos universitarios oficiales que habiliten para el ejercicio de la profesión de Maestro en Educación Primaria. BOE (29/12/2007), núm.312, referencia 22449, pp.53747-53750.

Ministerio de Educación y Ciencia (2007b). Real Decreto 1393/2007, de 29 de octubre, por el que se establece la ordenación de las enseñanzas universitarias oficiales. $\mathrm{BOE}$ (30/10/2007), núm.260, referencia 18770, pp.44037-44048.

Ministerio de Educación, Política Social y Deporte (2008). Real Decreto 1834/2008, de 8 de noviembre, por el que se definen las condiciones de formación para el ejercicio de la docencia en la educación secundaria obligatoria, el bachillerato, la formación profesional y las enseñanzas de régimen especial. BOE (28/11/2008), núm.287, referencia 19174, pp.47586-47591.

Ministerio de Educación, Ciencia y Deportes (2014). Real Decreto 126/2014, de 28 de febrero, por el que se establece el currículo básico de la Educación Primaria. BOE (01/03/2014), núm.52, referencia 2222, pp.19349-19420.

Pérez-Moreno, J., y Carrillo, C. (Eds.) (2019). El impacto de la educación musical escolar: una mirada retrospectiva. Barcelona: Octaedro.

Peñalba, A. (2017). La defensa de la educación musical desde las neurociencias. Revista Electrónica Complutense de Investigación en Educación Musical, 14, 109-127. doi:10.5209/RECIEM.54814

Peñalba, A. (2018). Claves para una educación musical temprana, creativa e inclusiva.

@Yurima Blanco García y Alicia Peñalba Acitores. The content of this article is the sole responsibility of the authors. The Revista Electrónica de LEEME and Universitat de València are not liable for any legal actions that may arise involving the article's content. Revista Electrónica de LEEME - Lista Electrónica Europea de Música en la Educación-. http://ojs.uv.es/index/php/LEEME/index ISSN: 1575-9563. Editores: Universidad de Valencia y Jesús Tejada. Visibilidad de esta revista: SCOPUS, Emerging Sources Citation Index (Clarivate), EBSCO, CINDOC (CSIC), Citefactor, COPAC, Dialnet, DICE (CSIC), DOAJ, e-revistas (CSIC), EBSCO Premier, ERIH+, Gale Cengage Learning, IN-RECS, IRESIE, LATINDEX, MIAR, OCLC Worldcat, RESH, REDIB, RILM Core Journals, SUDOC, ULRICHS. Esta revista es de acceso libre mediante licencia Creative Commons 4.0 CC by. Política de archivado: etiqueta verde SHERPA-ROMEO. 


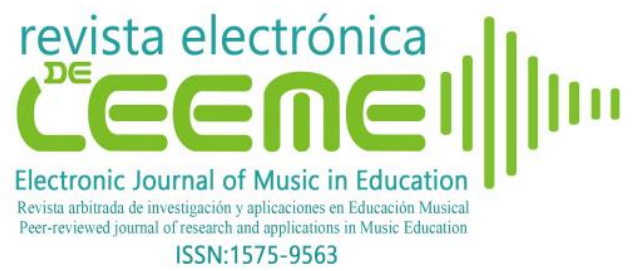

Tabanque: Revista Pedagógica, 31, 29-40. doi:10.24197/trp.31.2018.29-41

Profmus. Formación del Profesorado y Música en la Sociedad y en la Economía del Conocimiento (2017). Memoria del Proyecto I+D EDU2017-84782-P, financiado por el Ministerio de Economía y Competitividad del Gobierno de España. Recuperado de: http://profmus.ugr.es/

Reyes-López, M.L. (2010). Evaluación de los planes de estudio de formación del profesorado de educación musical: España. Profesorado. Revista de Currículum y Formación del Profesorado, 14(2), 67-81. Recuperado de: https://www.ugr.es/ recfpro/

Riaño, M.E. y Cabedo-Mas, A. (2013). La importancia del patrimonio musical en el aula. Eufonía: Didáctica de La Música, 58, 67-78. Recuperado de: https://core.ac.uk/download/pdf/80523127.pd

Rosa-Napal, F.C., Muñoz-Carril, P.C., González-Sanmamed, M. y Romero-Tabeayo, I. (2020). Musical expression in the training of future primary education teachers in Galicia. International Journal of Music Education, 33(4), 442-450. doi:10.1177/0255761420919566

Serrano, R.M. y Peñalba, A. (2019). Las características del profesorado como factores de impacto en la educación musical obligatoria. En J. Pérez-Moreno y C. Carrillo (Eds.), El impacto de la educación musical: una mirada retrospectiva (pp.101-116). Barcelona: Octaedro.

Small, C. (2003). Música, sociedad, educación. Madrid: Algibe.

Strauss, A. y Corbin, J. (1990). Basics of Qualitative Research: Grounded Theory Procedures and Techniques. Los Angeles: SAGE Publications Inc.

Tejada, J. y Pérez-Gil, M. (2016). Diseño y evaluación de un programa informático para la educación musical de maestros no especialistas. El caso de EMOLab. Revista Electrónica Complutense de Investigación en Educación Musical, 13, 22-49. doi:10.5209/reciem.52072

@Yurima Blanco García y Alicia Peñalba Acitores. The content of this article is the sole responsibility of the authors. The Revista Electrónica de LEEME and Universitat de València are not liable for any legal actions that may arise involving the article's content. Revista Electrónica de LEEME - Lista Electrónica Europea de Música en la Educación-. http://ojs.uv.es/index/php/LEEME/index ISSN: 1575-9563. Editores: Universidad de Valencia y Jesús Tejada. Visibilidad de esta revista: SCOPUS, Emerging Sources Citation Index (Clarivate), EBSCO, CINDOC (CSIC), Citefactor, COPAC, Dialnet, DICE (CSIC), DOAJ, e-revistas (CSIC), EBSCO Premier, ERIH+, Gale Cengage Learning, IN-RECS, IRESIE, LATINDEX, MIAR, OCLC Worldcat, RESH, REDIB, RILM Core Journals, SUDOC, ULRICHS. Esta revista es de acceso libre mediante licencia Creative Commons 4.0 CC by. Política de archivado: etiqueta verde SHERPA-ROMEO. 